\title{
A Dual/Single Wideband Switchable Metamaterial Absorber at Low Frequency
}

\author{
Liansheng Wang ( $\nabla$ wlswls1982@126.com ) \\ Sanya University \\ Dongyan Xia \\ Sanya University \\ Quanhong Fu \\ Northwestern Polytechnical University \\ Xueyong Ding \\ Sanya University

\section{Yuan Wang} \\ Sanya University
}

\section{Research Article}

Keywords: low frequency, switchable, dual wideband, single wideband, metamaterial absorber

Posted Date: January 3rd, 2022

DOI: https://doi.org/10.21203/rs.3.rs-1212897/v1

License: (c) (1) This work is licensed under a Creative Commons Attribution 4.0 International License. Read Full License 


\section{Abstract}

Based on PIN diode and resistive film, a dual/single wideband switchable metamaterial absorber at low frequency is presented in this paper. Its absorption is over $90 \%$ from $0.8 \mathrm{GHz}$ to $1.5 \mathrm{GHz}$ and from $4.2 \mathrm{GHz}$ to $5.2 \mathrm{GHz}$ while the PIN diode operates in forward biased condition. On the contrary, with the PIN diode acting in reverse biased condition, the above $90 \%$ absorption occurs from $1.1 \mathrm{GHz}$ to $3.2 \mathrm{GHz}$. The surface current distributions at the absorption frequencies are monitored to explain the reason of wideband absorption. The simulation results show that the absorption property of the metamaterial absorber is polarization-sensitive. The metamaterial absorber possesses the advantages of simple structure, wideband, dual/single band, and switchable performance.

\section{Introduction}

In the past ten years, metamaterials have attracted wide attention due to their remarkable electromagnetic properties that natural materials do not have ${ }^{[1-3]}$. At present, the research of metamaterials has made great progress on polarization conversion ${ }^{[4-6]}$, negative refraction ${ }^{[7-9]}$, perfect absorption ${ }^{[10-12]}$ and practical application ${ }^{[13-17]}$. As an important research field of metamaterials, metamaterial absorber can absorb incident waves at a specific frequency at the microwave, terahertz, infrared, and visible light bands ${ }^{[18-21]}$. The traditional electromagnetic absorption material has greatly restricted most practical applications due to the thickness restriction of $1 / 4$ of the wavelength while working at low frequency. However, the thickness of the metamaterial absorber can be very thin and mechanically flexible, and its absorption property can be optimized by designing its unit cell to have the advantages of wideband absorption, polarization-independent, and wide incident angle. The superior electromagnetic wave absorption performance of metamaterial absorbers benefits it with various important application prospects in the fields of detectors/emitters, hyperspectral sampling imaging, solar cells, plasma sensors, electromagnetic stealth, and so on.

In recent years, the research of metamaterial absorbers at low frequency has attracted wide attention. Due to the long wavelength of electromagnetic waves at low frequency and thickness limit of $1 / 4$ of the wavelength, traditional absorption materials at a low frequency generally becomes very thick and restrict many practical applications. However, the use of metamaterials permits the realization of absorbers to realize thin metamaterial absorbers with superior electromagnetic absorption properties. To contribute to the research of metamaterial absorber at low frequency, Liu et al. ${ }^{[22]}$ designed a metamaterial absorber based on circuit-resonant, showing absorption of over $90 \%$ from $840 \mathrm{MHz}$ to $960 \mathrm{MHz}$. Zuo et al. ${ }^{23}$ ] designed a three-layer metal square ring metamaterial absorber with an absorption greater than $90 \%$ from $300 \mathrm{MHz}$ to $3000 \mathrm{MHz}$. Nie et al. ${ }^{[24]}$ designed a composite metamaterial absorber with an absorption greater than $90 \%$ from $1 \mathrm{GHz}$ to $7 \mathrm{GHz}$.

The above-mentioned metamaterial absorber at low frequency has a single absorption band, whereas the multi-band switchable metamaterial absorber working at low frequency has more application prospects. 
At present, the PIN diode SMP1345-079LF is often used to achieve switchable metamaterial absorbers ${ }^{[25]}$. In this paper, we present a dual/single wideband switchable metamaterial absorber based on PIN diode SMP1345-079LF and resistive film at low frequency. When the PIN diode is forward biased, the absorption of the absorber is over $90 \%$ between $0.8 \mathrm{GHz} 1.5 \mathrm{GHz}$ and $4.2 \mathrm{GHz} 5.2 \mathrm{GHz}$; the absorption of the absorber stays above $90 \%$ from $1.1 \mathrm{GHz}$ to $3.2 \mathrm{GHz}$ when the PIN diode is reverse biased. It realizes multi-wideband absorption at low frequency based on the PIN diode at forward and reverse biased states. Compared with the reported metamaterial absorber at low frequency ${ }^{[26-31]}$, it has the advantages of having a simple structure, wideband, and dual/single band switchability with the potential application value on the fields of multi-spectral stealth, detection and communication.

\section{Model Design}

Our designed dual/single wideband switchable metamaterial absorber is shown in Figure 1. It is composed of five layers as shown in Figure 1(c), along the negative direction of the $z$-axis, they are the Ishaped copper resonant structure loaded with PIN diode followed by FR4 dielectric layer, copper rings loaded with resistive film, FR4 dielectric, and finally copper substrates as the backplane. The thicknesses of each layer are $0.02 \mathrm{~mm}, 2 \mathrm{~mm}, 0.02 \mathrm{~mm}, 2 \mathrm{~mm}$, and $0.02 \mathrm{~mm}$ respectively. The size parameters of unit cell are: $a=b=14 \mathrm{~mm}, c=d=12 \mathrm{~mm}, e=0.2 \mathrm{~mm}, r=3 \mathrm{~mm}, f=0.5 \mathrm{~mm}, g=0.5 \mathrm{~mm}$. The above-mentioned size parameters are obtained by parameter optimization based on the best absorption performance of the metamaterial absorber. The conductivity of the resistive film and copper is $5.871 \mathrm{mS} / \mathrm{m}$ and $5.7 \times 10^{\wedge} 7 \mathrm{~S} / \mathrm{m}$. The permittivity of the FR4 dielectric, and its tangent loss angle. The PIN diode used is SMP1345-079LF, and its equivalent circuit is shown in Figure 2. When the PIN diode is forward biased, it can be equivalent to a series connection of a small resistor and inductor which is induced by the wire at high frequency. On the contrary, with the PIN diode acting in reverse biased condition, it can be equivalent to a series connection of a very high resistor and capacitor. The equivalent circuit parameters for the $\mathrm{ON}$

state of PIN diode are $R_{\mathrm{on}}=50 \mathrm{hm}, L_{\mathrm{on}}=1.4 \mathrm{nH}$; while for the off state are $R_{\text {off }}=500000 \mathrm{hm}, C_{\text {off }}=0.4 \mathrm{pF}{ }^{[25,32]}$. The metamaterial absorber can be prepared through etching technology, where the practical difficulty lies in loading the resistive film.

The frequency-domain solver of the electromagnetic simulation software CST Microwave Studio is used to simulate the unit cell shown in Figure 1. During the simulation process, the wave vector lies along the $z$-direction, and the electric and magnetic fields are respectively along the $x$ and $y$ directions.

\section{Results And Discussion}

The bottom copper metal substrate of the unit cell makes its transmittance. The non-rotational symmetry of the unit cell converts part of the incident waves into cross-polarized waves. Therefore, the absorption can be calculated as, where, is the reflectivity of the co-polarized wave and is the reflectivity of the crosspolarized wave. According to the above formula, the $S$ parameters and calculated absorption of the metamaterial absorber with the PIN diode in both forward and reverse biased conditions is shown in 
Figure 3.The absorption of the metamaterial absorber exceeds $90 \%$ between $0.8 \mathrm{GHz} 1.5 \mathrm{GHz}$ and 4.2GHz 5.2G $\mathrm{Gz}$ when the PIN diode is forward biased, it, therefore, can realize dual wideband absorption. The absorption of the absorber is over $90 \%$ from $1.1 \mathrm{GHz}$ to $3.2 \mathrm{GHz}$ when the PIN diode is reverse biased. Therefore, altogether the metamaterial absorber has the dual/single wideband switchable property.

According to the calculated formula of absorption, $A(\omega)=1-R_{x, x}(y \cdot y)-R_{x, y}(y \cdot x)\left(R(\omega)=\left|S_{11}(\omega)\right|^{2}\right.$ is the reflectivity), the perfect absorption can be realized when the reflectivity $R(\omega)=\left|S_{11}\right|^{2}=0$. The condition for achieving zero reflectivity $\left|S_{11}\right|^{2}=0$ is that the relative impedance $\left(Z(\omega) / Z_{0}, Z(\omega)\right.$ is the input impedance of metamaterial absorber, $\mid Z_{0}$ is the input impedance of free space)must be equal to one. Figure 4 shows the relative impedance calculated by using the scattering parameter method ${ }^{[33]}$ for both cases when the PIN diode is forward biased and reverse biased. It can be seen from Figure 4(a) that the real part of the relative impedance is close to one between $0.8 \mathrm{GHz} \sim 1.5 \mathrm{GHz}$ and $4.2 \mathrm{GHz} \sim 5.2 \mathrm{GHz}$ when the PIN diode is forward biased. At this condition, the metamaterial absorber has less reflection of the incident waves, and the absorption becomes high. The negative imaginary part of the relative impedance indicates that the metamaterial absorber undergoes a large amount of loss to the incident waves. It can be seen from Figure 4(b) that the relative impedance of metamaterial absorber with that of the free space is also close to one from $1.1 \mathrm{GHz}$ to $3.2 \mathrm{GHz}$ when the PIN diode is reverse biased, it achieves the precondition of perfect absorption.

In order to explore the wideband absorption mechanism of the metamaterial absorber with the PIN diode in forward biased condition in a further step, the surface current distributions of the metamaterial absorber at $1 \mathrm{GHz}, 1.2 \mathrm{GHz}, 4.5 \mathrm{GHz}$, and $4.8 \mathrm{GHz}$ are monitored, as shown in Figure 5, Figure 6, Figure 7 and Figure 8. It can be seen from Figure 5 and Figure 6 that the surface currents excited by the incident waves on the left and right sides of the metal copper ring are parallel and directed upwards. This parallel surface current causes the alternative accumulation of charge on the upper and lower parts of the copper ring, thereby generating electric dipole resonance ${ }^{[34]}$. The surface current on the l-shaped metal resonance structure and the metal copper ring is in the opposite direction to the surface current on the metal copper substrate. This surface current in the opposite direction forms a current loop that generates magnetic resonance ${ }^{[34]}$. The magnetic and electric resonance formed at $1 \mathrm{GHz}$ and $1.2 \mathrm{GHz}$ loses the energy of the incident wave and offers perfect absorption. Therefore, the absorption peaks at $1 \mathrm{GHz}$ and 1.2 $\mathrm{GHz}$ originate from the electromagnetic resonance generated by the metamaterial absorber at the action of incident waves. It can be seen from Figure 7 and Figure 8 that the parallel surface currents on the left and right sides of the metal copper ring produce electric dipole resonance ${ }^{[34]}$. On the contrary, the surface current of the l-shaped metal resonance structure and the metal substrate works in the opposite direction to the metal copper ring, which gives rise to magnetic resonance ${ }^{[34]}$. Therefore, the absorption peaks at $4.5 \mathrm{GHz}$ and $4.8 \mathrm{GHz}$ also originate from the electromagnetic resonance generated by the 
metamaterial absorber at the action of incident waves. The overlay of different absorption frequencies leads to wideband absorption.

Figure 9 and Figure10 show the surface current distributions of metamaterial absorber at $2 \mathrm{GHz}$ and $2.5 \mathrm{GHz}$ when the PIN diode is reverse biased. It can be seen from Figure 9 and Figure 10 that the parallel surface currents excited by the incident waves on the left and right sides of the metal copper ring cause the electric dipole resonance ${ }^{[34]}$. On the other hand, the surface current of the l-shaped metal resonant structure and the metal copper ring works in the opposite direction to the metal substrate, this kind of surface current distribution in the opposite direction forms magnetic resonance ${ }^{[34]}$. Therefore, the absorption peaks at $2 \mathrm{GHz}$ and $2.5 \mathrm{GHz}$ with the PIN diode in reverse biased condition originate from the electromagnetic resonance generated by the metamaterial absorber at the action of incident waves. The reason for wideband absorption stems from the overlay of different absorption frequencies.

In order to study the relationship of the absorption property of metamaterial absorber with the polarization angle of the incident wave, the absorption of metamaterial absorber based on the the polarization angle when the PIN diode is forward biased and reverse biased are calculated, as shown in Figure 11. It can be seen from Figure 11 that the bandwidth of metamaterial absorber with the absorption over $90 \%$, gradually decreases with the increasing of the polarization angle, which indicates that the absorption of metamaterial absorber is polarization-sensitive due to the non-rotational symmetry of unit cell.

In order to study the relationship of the absorption property of the metamaterial absorber with incident angle, the absorption of the metamaterial absorber under different incident angles with the PIN diode is forward biased, and reverse biased conditions are calculated, as shown in Figure 12 and Figure 13. It can be seen from Figure 12 that when the PIN diode is forward biased, the absorption of the metamaterial absorber between $0.8 \mathrm{GHz} \sim 1.5 \mathrm{GHz}$ and $4.2 \mathrm{GHz} 5.2 \mathrm{GHz}$ decreases gradually with the increasing of the incident angle at TE mode. The absorption of the metamaterial absorber from $1.1 \mathrm{GHz}$ to $3.1 \mathrm{GHz}$ stays over $90 \%$ due to the non-rotational symmetry of the unit cell and the absorption gradually decreases with the increasing of the incident angle at TM mode. Figure 13 shows that the absorption of the metamaterial absorber from $1.1 \mathrm{GHz}$ to $3.2 \mathrm{GHz}$ decreases gradually with the increasing of the incident angle at TE and TM mode when the PIN diode is reverse biased.

\section{Conclusion}

The electromagnetic waves at low frequencies have important applications in long-distance wireless communication, monitoring, and early warning. Due to the long wavelength of the electromagnetic waves at low frequency and the thickness restriction of $1 / 4$ wavelength, the traditional absorbing materials at a low frequency generally become very thick, which greatly limits its practical applications. It has great significance as metamaterial absorbers to realize advanced and efficient metamaterial absorbers at low frequency without being restricted by the thickness of $1 / 4$ wavelength. In this paper, we present a dual/single wideband switchable metamaterial absorber at a low frequency based on PIN diodes and 
resistive films. The absorption of the metamaterial absorber between $0.8 \mathrm{GHz} 1.5 \mathrm{GHz}$ and 4. $2 \mathrm{GHz} 5.2 \mathrm{GHz}$ is over $90 \%$ when the PIN diode is forward biased, and its absorption is over $90 \%$ from $1.1 \mathrm{GHz}$ to $3.2 \mathrm{GHz}$ when the PIN diode is reverse biased. The surface current distribution at the absorption frequency indicates that the electromagnetic resonance generated under the action of the incident wave causes the absorption of the incident wave, and the wideband absorption originates from the overlay of different absorption frequencies. It has the advantages of simple, wideband, and dual/single wideband switchable, and has potential application value on electromagnetic shielding and electromagnetic stealth at low frequency.

\section{Declarations}

\section{Acknowledgement}

This work is supported by Hainan Provincial Natural Science Foundation of China (Granted number:620MS062)

\section{Data availability}

The data that support the findings of this study are available from the corresponding author upon reasonable request.

\section{References}

1. J. Grant, Y. Ma, S. Saha, L. B. Lok, A. Khalid, and D. R. S. Cumming,Opt. Lett. 36(8),1524-1526(2011).

2. L.Ju, B.S.Geng, J.Horng, C. Girit, M. Martin, H.Zhao, H.A. Bechtel, X.G. Liang, A.Zettl, Y.R.Shen, and F.Wang, Nat. Nanotechnol. 6(10),630-634(2011).

3. X.Y.He, Z.Y.Zhao, and W. H. Shi,Opt. Lett. 40(2),178-181(2015).

4. J.Hu, G. Q.Hu, and Z. C. Hao,IEEE Access.6,6130-6137(2018).

5. X.Gao, X.Han, W.P.Cao, H.O.Li, H. F. Ma, and T.J.Cui,IEEE T. Antenn. Propag. 63(8),3522-3530(2015).

6. Z.Wei, C.Yang, Y. Fan, Y. Xing, and H. Li, Appl. Phys. Lett. 99(22),221907(2011).

7. D.R.Smith,W.J.Padilla,D.C.Vier,S.C.Nemat-Nasser,andS.Schultz,Phys.Rev.Lett.84(18),41844187(2000).

8. V.Yannopapas,J. Phys-Condens. Mat. 18(29), 6883-6890(2006).

9. A. N. Grigorenko,A. K. Geim,H. F. Gleeson,Y. Zhang,A. A. Firsov,I. Y. Khrushchev,and J. Petrovic,Nature. 438,335-338(2005).

10. J.W.Xie, S. Quader, F.J.Xiao, C.He, X.L.Liang, J.P.Geng, R.H.Jin, W.R.Zhu, and I.D.Rukhlenko, IEEE Trans. Antennas Propag. 18 (3),536-540(2019).

11. J.Zhang, Z.F.ZLi, L.D.Shao, and W.R.Zhu,Carbon.176,374-382(2021).

12. S. Quader, M.R. Akram, F.J.Xiao, and W.R. Zhu,Journal of Optics.22(9),095104(2020). 
13. H.X Xu, G.M Wang, M.Q Qi, Y.Y Lv, and X Gao, Appl.Phys.Lett.102,193502(2013)

14. S. Roy, K. L Baishnab, and U Chakraborty, Progress in electromagnetics research C. 83, 33-44(2018).

15. H.X Xu, G.M Wang, M.Q Li, L.M Li,and T.J Cui, Adv.Opt.Mater.1,495-502(2013).

16. S Roy, U. Chakraborty, IET Communications.12(12),1448-1453(2018).

17. M Bakir, M. Karaaslan, $O$ Altintas, M Bagmanc, V Akdogan, and F Temurta, Int J Microw Wireless Tech. 10,67-76(2018).

18. N. Liu, M. Mesch, T. Weiss, M. Hentschel, and H. Giessen,Nano Lett.10(7),2342-2348(2010).

19. K.Iwaszczuk,A.C.Strikwerda,K.Fan,X.Zhang,R.D.Averitt,andP.U.Jepsen,Opt. Express. 20(1), 635-643 (2012).

20. T.Hu, C.M. Bingham, A.C. Strikwerda, D. Pilon, D. Shrekenhamer, N. I. Landy, K. Fan, X. Zhang, W. J. Padilla, and R. D. Averitt, Phys. Rev. B.78,241103(2008).

21. M. Diem, T. Koschny, and C.M.Soukoulis,Phys. Rev. B.79,033101(2009).

22. X.C.Xiao,W.W.Zhang,W.X.Hu,M.Q.Jia,X.X.He,and D.Ben, Journal of Nanjing University of Aeronautics \& Astronautics.51(6),864-869(2019).

23. W.Q.Zuo, Y.YangY, and X.X.He,Hefei:2015 National Conference on Microwave and Millimeter Waves. 1670-1673(2015).

24. Y.Nie, Y.Z.Cheng, and R.Z.Guo,Chin. Phys. B. 22(4),44102(2013).

25. R.Zhao,B.Y.Zhao,F.J.Zhao,H.Chong,and W.R.Zhu,IEEE Access.7,55518-55523(2019).

26. J.C. Mou, Z.X. Shen, Sci. Rep. 7, 6922(2017).

27. J.C. Mou, Z.X. Shen, IEEE Trans. Antennas Propag. 65, 696-704(2016)

28. M. D. Banadaki, A .A. Heidari, and M. Nakhkash, IEEEAntennas Wirel. Propag. Lett. 17, 205-208 (2017)

29. W. Li, J. Wei, W. Wang, D.W. Hu, Y.K. Li, and J.G. Guan, Mater.Des. 110, 27-34(2016)

30. Y. Z. Cheng, Y. Wang, Y. Nie, D. H. Zheng, R. Z. Gong, X. Xiong, and X. Wang, Acta Phys. Sin. 61,134102 (2012)

31. W.C.Li, Q. Liu, L.W.Wang, Z.Z. Zhou, J.W. Zheng, Y. Ying, L. Qiao, J. Yu, X.J Qiao, and S.L Che, AIP Adv. 8, 015318(2018)

32. Pin Diode SMP1215 Series Datasheet, Skyworks Solutions,Woburn, MA,USA, 2011

33. D.R.Smith, S.Schultz, Phys.Rev.B. 65,195104(2002).

34. X.P.Shen, T.J.Cui, and J.X.Ye, Acta Physica Sinica. 61(5),058101(2012).

\section{Figures}




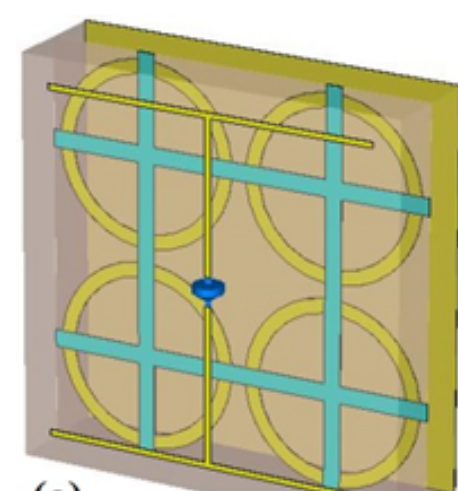

(a)
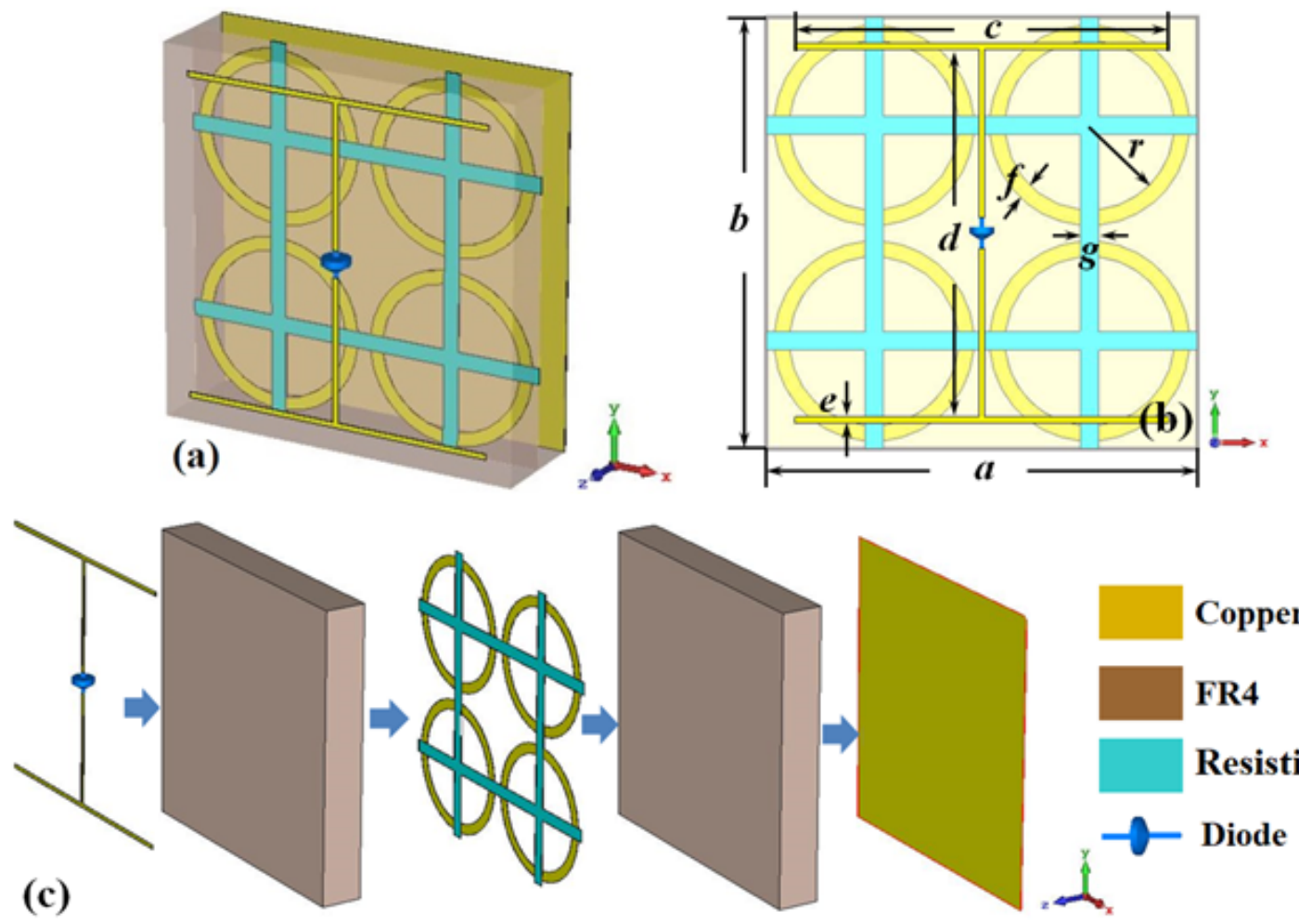

Figure 1

The diagram of the absorber, (a) perspective view; (b) front view; (c) layered-structure

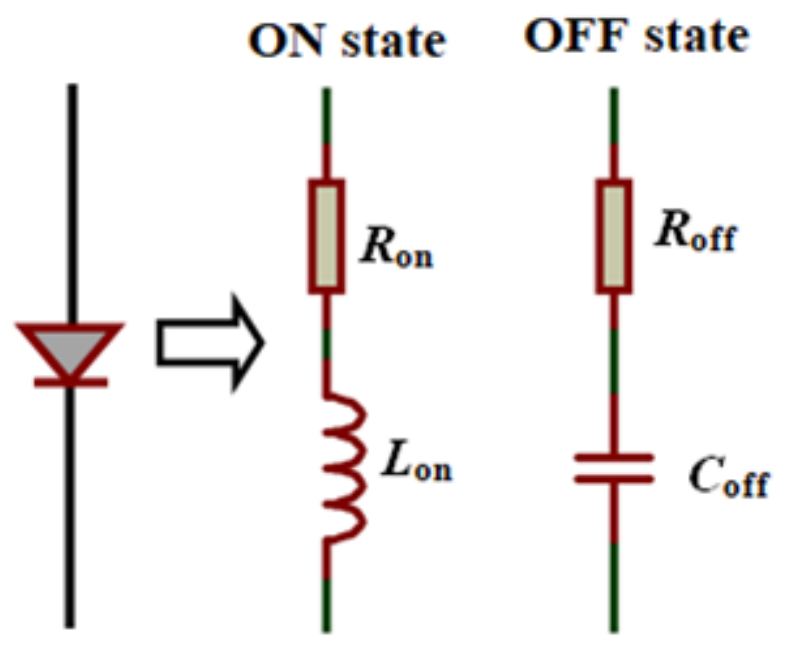

Figure 2

The equivalent circuit of the PIN diode for on and off states 

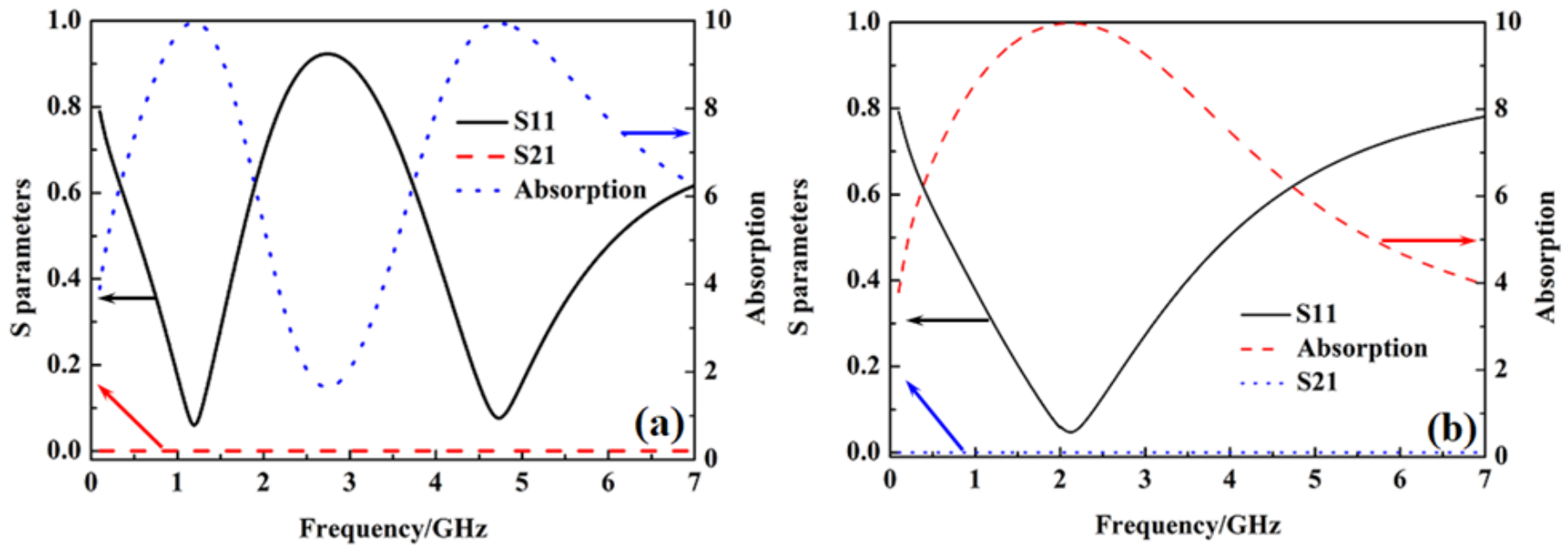

Figure 3

The absorption with the PIN diode ON and OFF state,(a) ON state,(b)OFF state
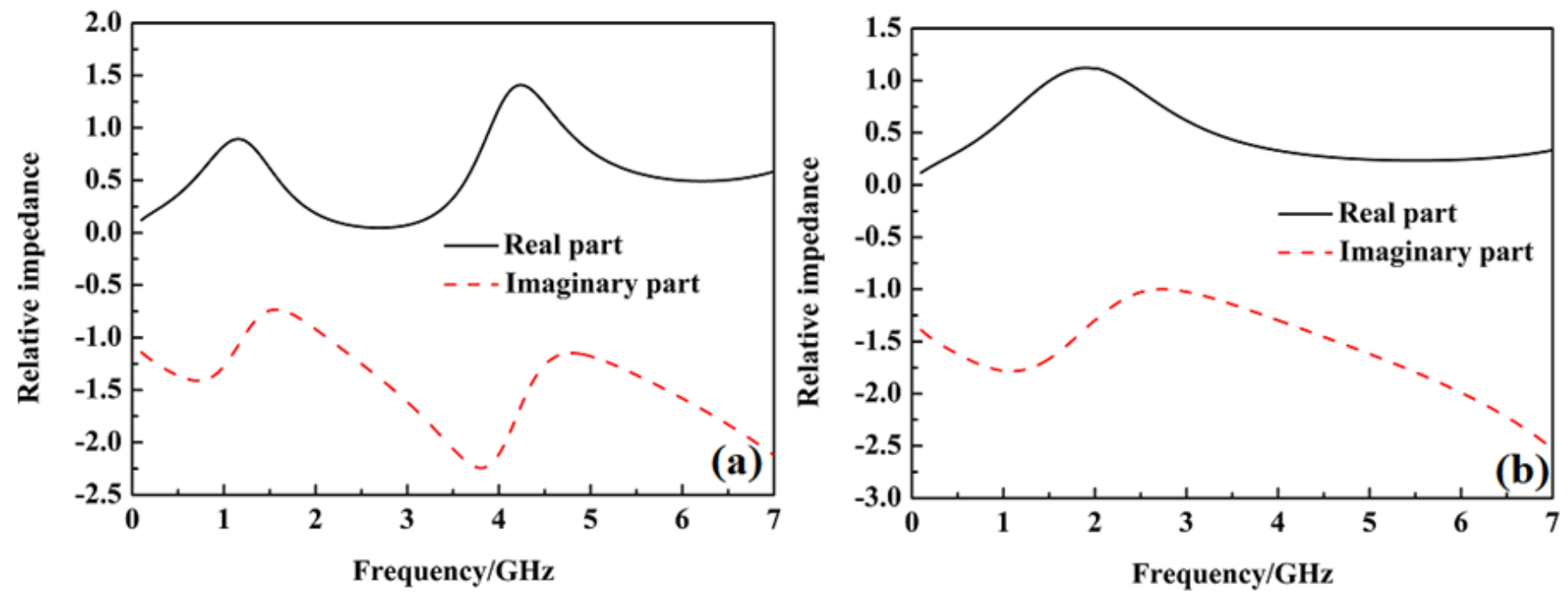

Figure 4

The relative impedance, (a) PIN diode for ON state; (b) PIN diode for OFF state 


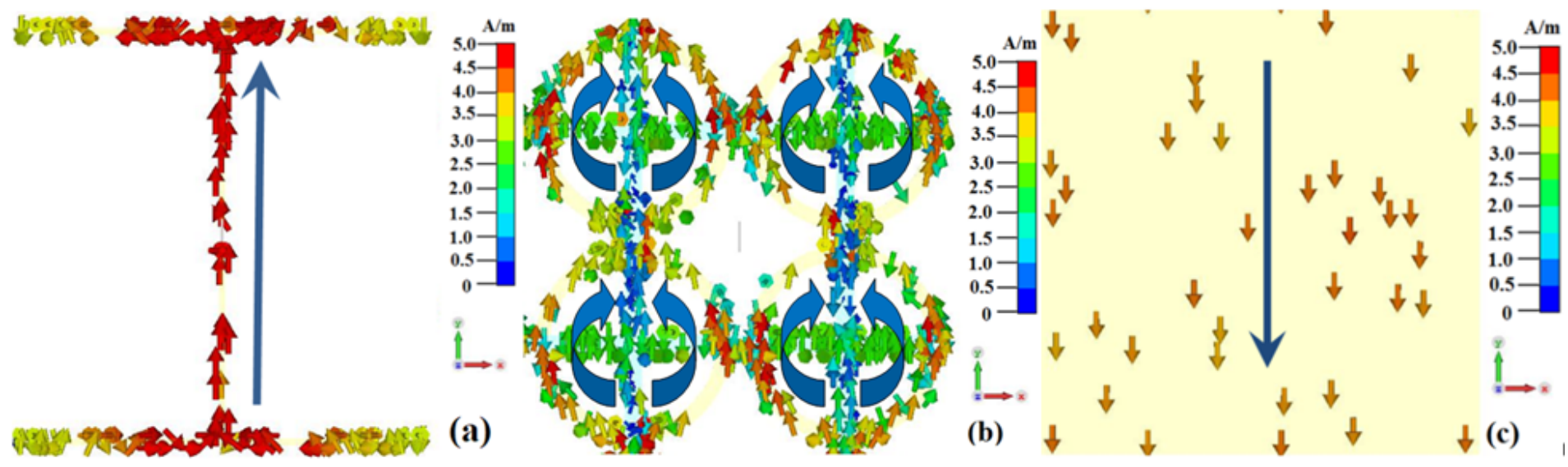

Figure 5

The surface current distribution with the PIN diode in forward biased condition at $1 \mathrm{GHz}$, (a) I-shaped metal resonant structure ;(b) metal copper ring;(c) copper substrate

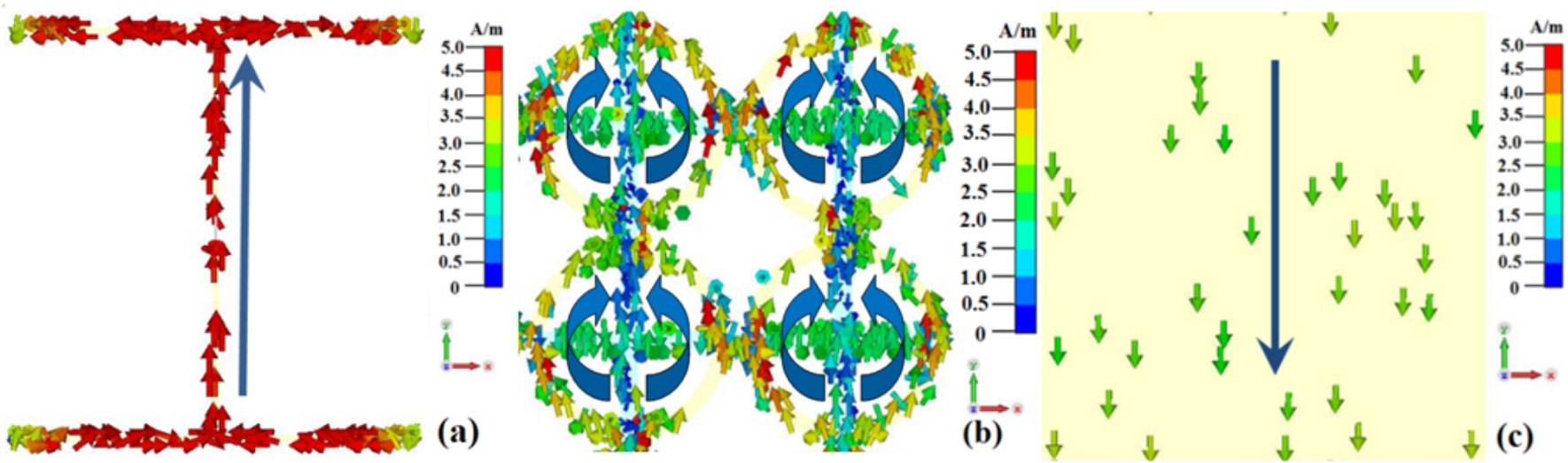

Figure 6

The surface current distribution with the PIN diode in forward biased condition at $1.2 \mathrm{GHz}$, (a) I-shaped metal resonant structure;(b) metal copper ring;(c) copper substrate

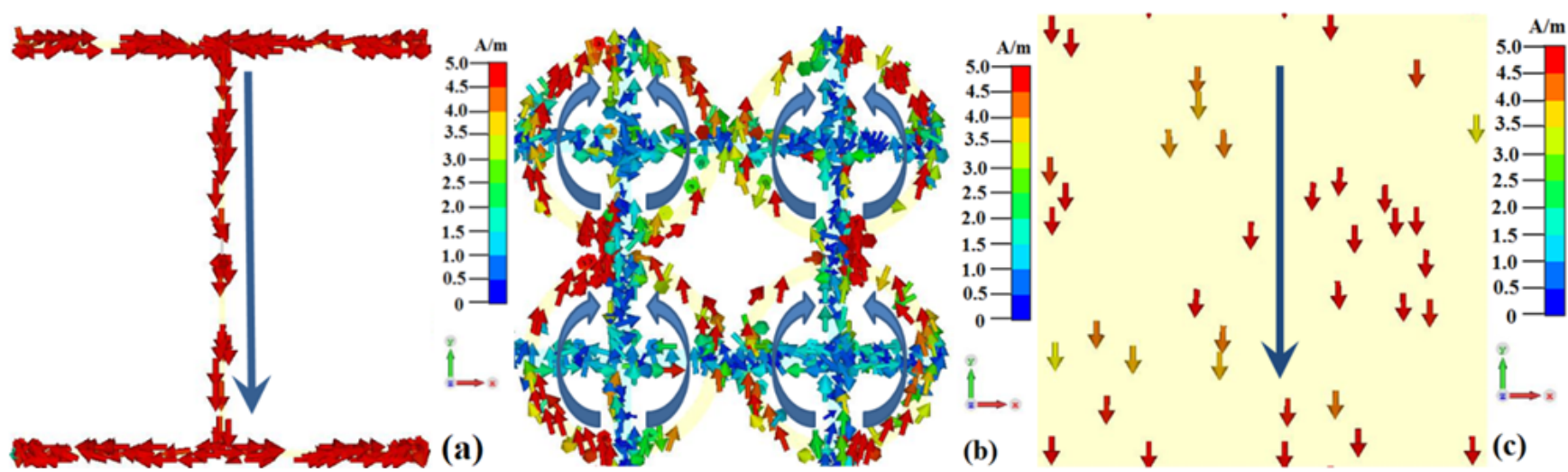

Figure 7 
The surface current distribution with the PIN diode in forward biased condition at $4.5 \mathrm{GHz}$, (a) I-shaped metal resonant structure ;(b) metal copper ring;(c) copper substrate

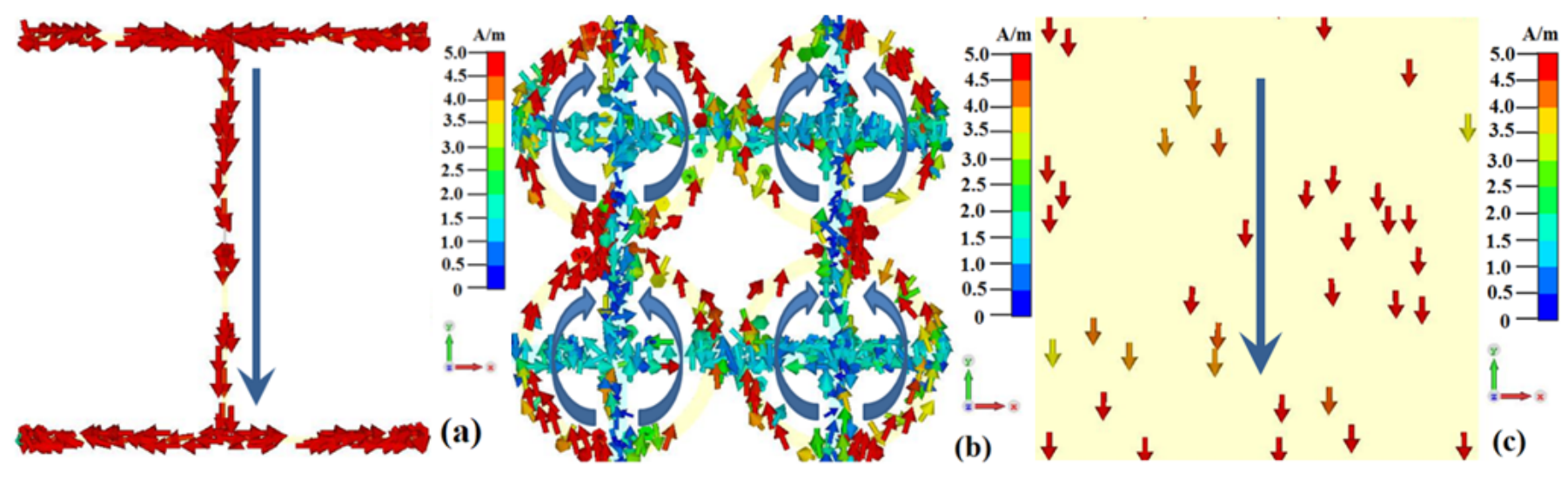

Figure 8

The surface current distribution with the PIN diode in forward biased condition at 4.8GHz, (a) I-shaped metal resonant structure;(b) metal copper ring;(c) copper substrate

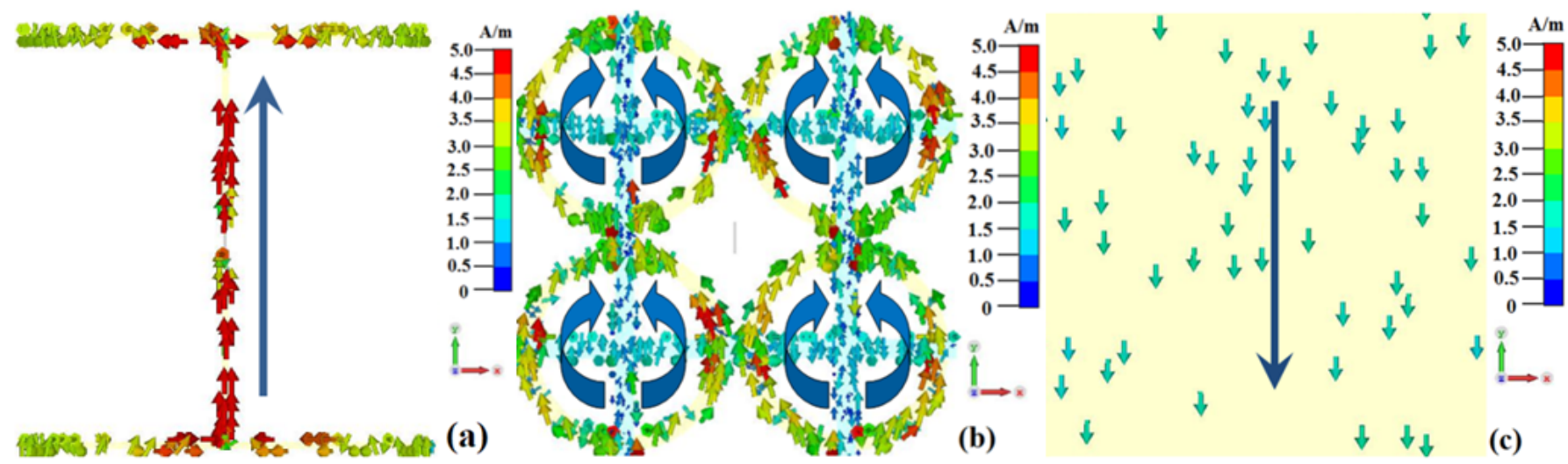

Figure 9

The surface current distribution with the PIN diode in reverse biased condition $2 \mathrm{GHz}$, (a) I-shaped metal resonant structure; (b) metal copper ring; (c) copper substrate 


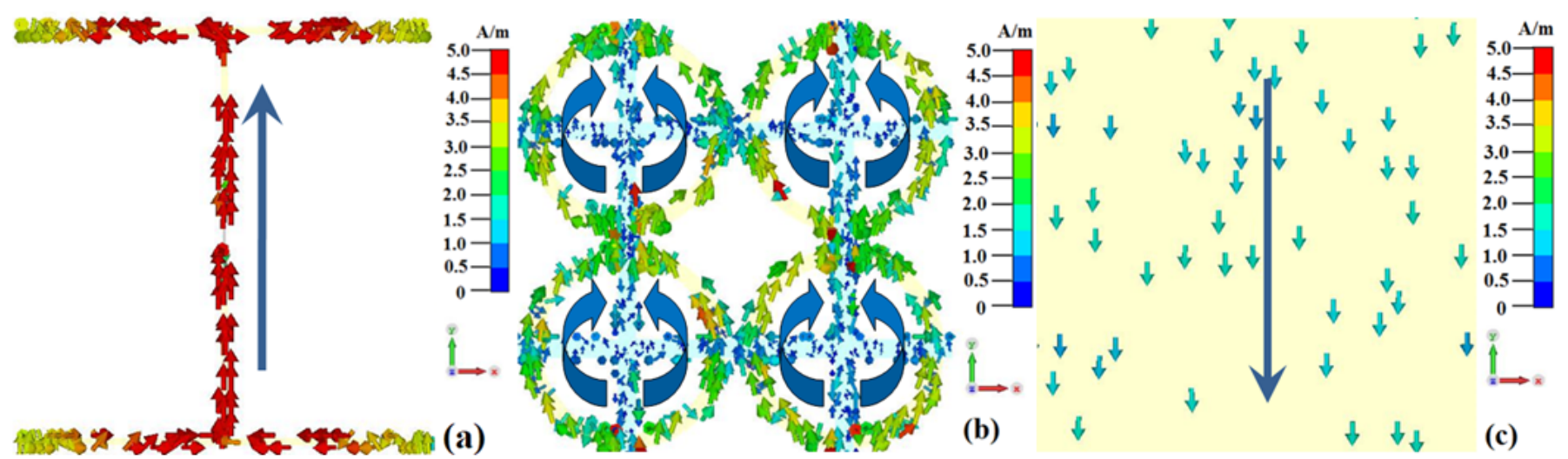

Figure 10

The surface current distribution with the PIN diode in reverse biased condition at $2.5 \mathrm{GHz}$, (a) I-shaped metal resonant structure; (b) metal copper ring; (c) copper substrate
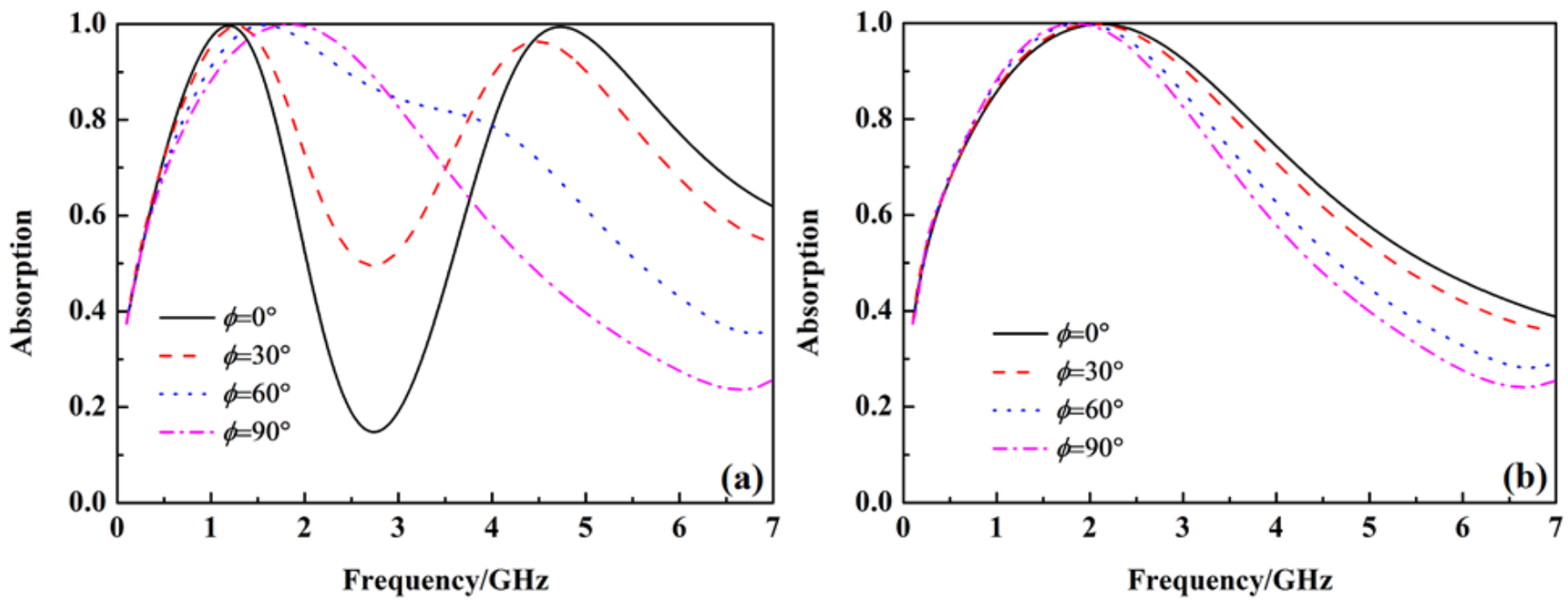

Figure 11

The absorption as functions of polarization angles when the PIN diode is forward biased and reverse biased, (a) ON state; (b) OFF state 

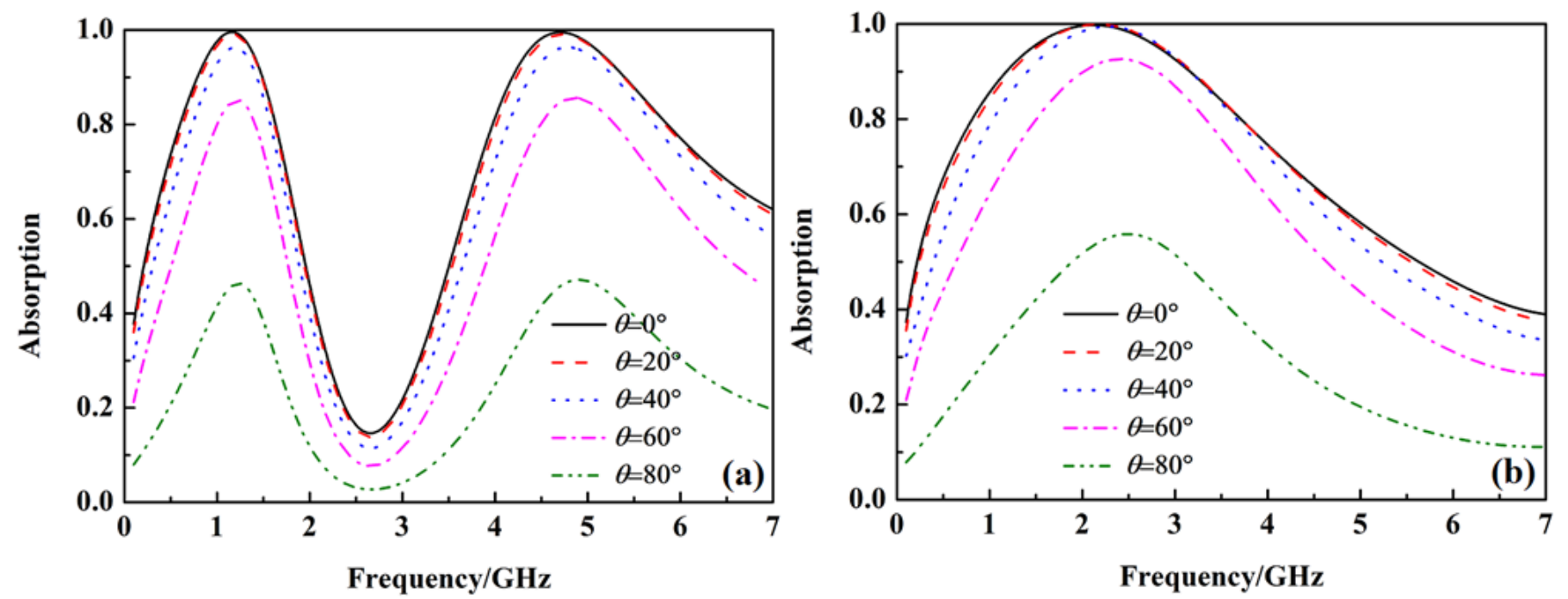

Figure 12

The absorption as functions of incident angles when the PIN diode is forward biased, (a) TE mode; (b) TM mode.
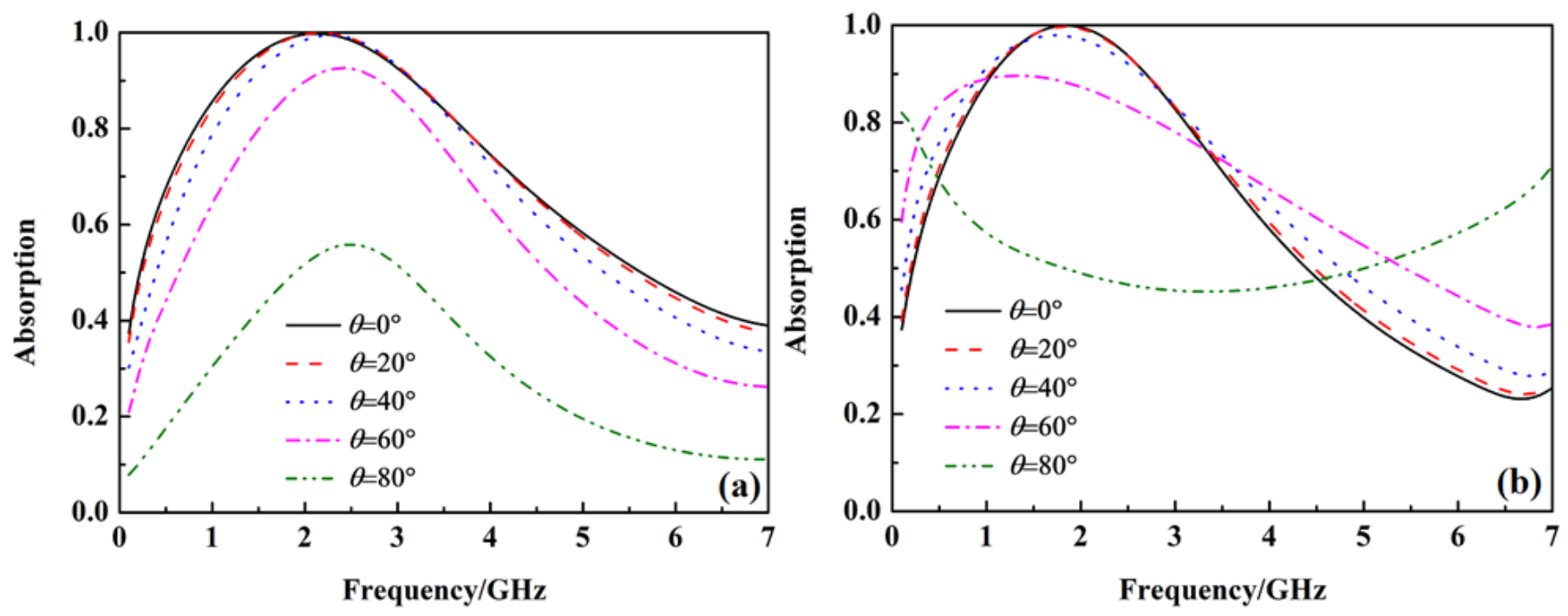

Figure 13

The absorption of the metamaterial absorber under different incident angles when the PIN diode is reverse biased,(a) TE mode; (b)TM mode. 